\title{
Model Identification of a Small Fully-Actuated Aquatic Surface Vehicle Using a Long Short-Term Memory Neural Network
}

\author{
Document Version \\ Accepted author manuscript
}

Link to publication record in Manchester Research Explorer

Citation for published version (APA):

Dimitrov, M., Groves, K., Howard, D., \& Lennox, B. (2021). Model Identification of a Small Fully-Actuated Aquatic Surface Vehicle Using a Long Short-Term Memory Neural Network. Paper presented at IEEE ICRA 2021, Xi'an, China.

\section{Citing this paper}

Please note that where the full-text provided on Manchester Research Explorer is the Author Accepted Manuscript or Proof version this may differ from the final Published version. If citing, it is advised that you check and use the publisher's definitive version.

\section{General rights}

Copyright and moral rights for the publications made accessible in the Research Explorer are retained by the authors and/or other copyright owners and it is a condition of accessing publications that users recognise and abide by the legal requirements associated with these rights.

\section{Takedown policy}

If you believe that this document breaches copyright please refer to the University of Manchester's Takedown Procedures [http://man.ac.uk/04Y6Bo] or contact uml.scholarlycommunications@manchester.ac.uk providing relevant details, so we can investigate your claim.

\section{OPEN ACCESS}




\title{
Model Identification of a Small Fully-Actuated Aquatic Surface Vehicle Using a Long Short-Term Memory Neural Network
}

\author{
Marin Dimitrov $^{1}$, Keir Groves ${ }^{1}$, David Howard ${ }^{2}$ and Barry Lennox ${ }^{1}$
}

\begin{abstract}
A long short-term memory neural network is used to provide a system model that captures the temporal-dynamics of a holonomic, fully-actuated aquatic surface vehicle. As is true in many fields, new developments in robotics often are made in simulation first before being applied to real systems. To simulate an aquatic or aerial robot, a dynamic system model of the robot is required. The more representative the dynamic model is of the real robot, the smaller the simulation-to-reality gap becomes. The performance of the neural network is compared against a classical parametric model, where coefficients of the parametric model were identified using the same data that was used to train the neural network. The results show that the neural network consistently outperforms the classical parametric model and significantly reduces the error between real velocities and estimated velocities. The neural network also demonstrated the ability to capture complex hydrodynamic effects that were not captured in the parametric model. In addition to the performance improvements, the neural network method can be easily adapted to similarly actuated aquatic vehicles by simply retraining, whereas the classical approach would require manual selection of new equation terms. The neural network model that was created has been used in a vehicle simulation and is presently being used as a research tool.
\end{abstract}

\section{INTRODUCTION}

\section{A. Motivation}

Aquatic surface vehicles can be useful in a range of scenarios such as ship hull inspection, water quality monitoring or nuclear fuel storage pool inspection. Two main factors determine the possible application areas for an aquatic surface vehicle: size and the accuracy with which the vehicle can hold a trajectory or position, here termed control tracking accuracy. For example, a $4.8 \mathrm{~m}$ long autonomous surface vehicle (ASV) that used GPS localisation and had control tracking accuracy of $1 \mathrm{~m}$ was successfully used to measure a range of water quality properties in a lake [1]. However, this vehicle would not be suitable for use in a smaller confined environment, such as a nuclear fuel storage pool, where a vehicle must be able to control its path and position with much higher accuracy. The need for smaller surface vehicles with high tracking accuracy led researchers at the University of Manchester to develop MallARD (Small Autonomous Robotic Duck) [2]. MallARD is an ASV that was primarily designed to operate in spent nuclear fuel storage pools and achieves a control tracking accuracy of less than $1 \mathrm{~cm}$ using a 2D LiDAR as its main sensor. Although designed for use in fuel storage pools, MallARD can be used for

1 Department of Electrical and Electronic Engineering, University of Manchester, UK; contact keir.groves@manchester.ac.uk

2 Robotics and Autonomous Systems Group, Data61, CSIRO, Australia inspection and monitoring purposes in most confined space water applications e.g., process vessels or water storage facilities.

To speed-up development of MallARD, it is useful to have a computer simulation of the robot. Simulation of a complete robot and how it interacts with its surroundings is now commonplace and new innovations are usually tested in simulation before being implemented on the actual system [3].

As well as purely software simulation, a unique robot, termed here a hardware simulation, has been produced that facilitates development of ASVs without the need for a pool [4]. The hardware simulation is a holonomic ground robot that can mimic the physical behaviour of the true target robot, using real sensors and identical software configurations. The hardware simulation is used extensively for development of the MallARD platform.

The hardware simulation is a more realistic alternative that is preferable when testing on the actual robot is expensive or difficult, which is often the case for aquatic robots as they require extended access to pool facilities. Furthermore, connectivity between system components can be validated and electronics tested. An example of a hardware simulation of the El-MallARD robot [4]. El-MallARD is an omnidirectional ground vehicle capable of mimicking the physical behaviour of an aquatic surface vehicle, if a dynamic model is provided.

For both hardware and software simulation, a dynamic system model is required. The accuracy of the model in terms of its ability to predict the physical behaviour of the real robot is important for a number of reasons. First, a highly accurate model allows more testing and development to take place in simulation, which can save time and cost. Second, if the dynamic model is very representative of the real system then the transition between running software in simulation and on the real robot is easier and less prone to error. Finally, the system model can be used as an aid to the robots localisation package, e.g. [5] and [6]. If the model can provide accurate velocity predictions, these can be fused with measurements from other sensors to improve precision of the location estimate [7].

In this work, two different system models of MallARD are compared in terms of their performance, one using a classical parametric approach based on a theoretical model and the other using modern recurrent neural networks. The parametric model and method of identifying parameters has been previously covered in-depth in a previous article by the authors [8]. A key problem that was identified in the 
author's previous work is the choice of appropriate equation terms for inclusion in the model. This is a common problem associated with parametric system models. It can be difficult to correctly include and identify coefficients for complex and often coupled hydrodynamic model parameters. If salient parameters are missed, the model will lose accuracy during certain manoeuvres [9]. Using an appropriate, trained neural network in place of the classical, theoretical model removes the need for manual selection of appropriate theoretical model parameters. This has the potential to provide a more robust and accurate model that is easier to train or identify than a classical parametric model. Therefore, a temporally sensitive long short-term memory (LSTM) neural network was used to provide the dynamic model, as it overcomes the identified weaknesses of the aforementioned parametric approach.

\section{B. Literature review}

Within the literature there are various contributions to parametric modelling of both surface and underwater vessels. In their paper [10], Eriksen and Breivik presented a parametric model and a control system for a high-speed aquatic surface robot. The vehicle was $8.45 \mathrm{~m}$ long, $2.71 \mathrm{~m}$ wide and could reach speeds of up to $18 \mathrm{~m} / \mathrm{s}$. A 2 DOF model was implemented because the vehicle was underactuated and operated only in surge and yaw. For parameter identification a weighted linear least squares method was used. Li et al. [11] described the modelling of an unmanned surface vessel with rudderless double thrusters. The vehicle had a catamaran structure and was able to reach up to 1.5 $\mathrm{m} / \mathrm{s}$. Due to the low speed, the authors neglected nonlinear drag from the dynamic equation and used linear least squares for parameter identification. Sonnenburg and Woolsey [12] presented a summary of the system identification techniques developed for use on aquatic surface robots covering both parametric and non-parametric approaches.

Although there is a variety of examples of parametric system modelling of aquatic vehicles within the literature, there are significantly fewer contributions related to modelling using neural networks. The example that is most similar to the approach of the authors is presented by Woo et. al. [13], who developed a system model that combined a classical parametric approach with an LSTM neural network to account for nonlinearities. The addition of the neural network to the parametric model led to improvements in model accuracy. The vehicle identified in [13] was significantly different to MallARD, being underactuated and much larger in size. Moreover, in [13] the LSTM network was used to augment a parametric model, meaning two models were required and equation terms still needed to be manually selected. In this paper an LSTM network is used in isolation from the parametric model, removing the need to manually select model parameters.

Neural networks have not been widely used for modelling of aquatic surface vehicles. However, there is a large number of works related to neural networks for dynamic system modelling. For instance, in [14] an artificial neural network was used to model a 2D damper bearing and the network outperformed a theoretical model that was derived from the fluid mechanics of the damper. Recently, in [15] a combination of neural networks was used for dynamic modelling of a legged robot, specifically representing the actuators with an identified model based on a feedforward neural network. This allowed useful behaviours to be learned in simulation, and sidestep the usual 'reality gap' issues when transferring back to the real robot as motor dynamics were properly represented in the simulation and captured from real-world data.

One of the first papers to model vehicle dynamics with neural networks was applied to identify dynamic models of a helicopter [16], and subsequently used those models to perform aerobatic manoeuvres. The neural network showed significant improvements over other state-of-the-art approaches, especially where dynamics were highly nonlinear and difficult to obtain using standard approaches.

Other examples of nonlinear system identification include the use of convolutional networks [17], or combination with randomized algorithms [18], however, the authors were motivated to use LSTM networks due to their temporal sensitivity and ability to represent time-series dynamics over multiple temporal resolutions.

\section{Contribution}

The main contributions of this paper are as follows:

1) Development of an LSTM recurrent neural network for modelling the dynamic behaviour of a small, fully actuated aquatic surface vehicle;

2) A performance comparison between the LSTM neural network model and a classical parametric model with empirically identified coefficients.

\section{HARDWARE ARCHITECTURE}

\section{A. Hardware components}

MallARD, presented in Figure 1 is a holonomic aquatic surface vehicle, designed to operate at low speeds (0$1 \mathrm{~m} / \mathrm{s}$ ) in confined spaces. It consists of two pontoons of length $500 \mathrm{~mm}$, where all the electronic components are encapsulated. When running in manual control, MallARD receives analogue control inputs from a joystick which are translated to thruster outputs. The joystick is connected to a control laptop that wirelessly communicates with an on-board computer. The commands in surge, sway and yaw are transformed into PWM inputs for the individual thrusters using the thrust allocation scheme (Section II-B). PWM signals are sent from a connected microcontroller to the electronic speed controllers which are directly connected to the brushless motors of the thrusters. All systems onboard are powered by an $8 \mathrm{Ah}, 22.2 \mathrm{v}$, 6s Lipo battery. The on-board computer processes data from a 2D LiDAR and runs a SLAM algorithm to estimate the pose of the robot [19]. In addition to manual control, MallARD can follow predefined trajectories and station keep under closed loop trajectory control. A more detailed system design as well as full description of the control systems implemented can be found in [2]. 


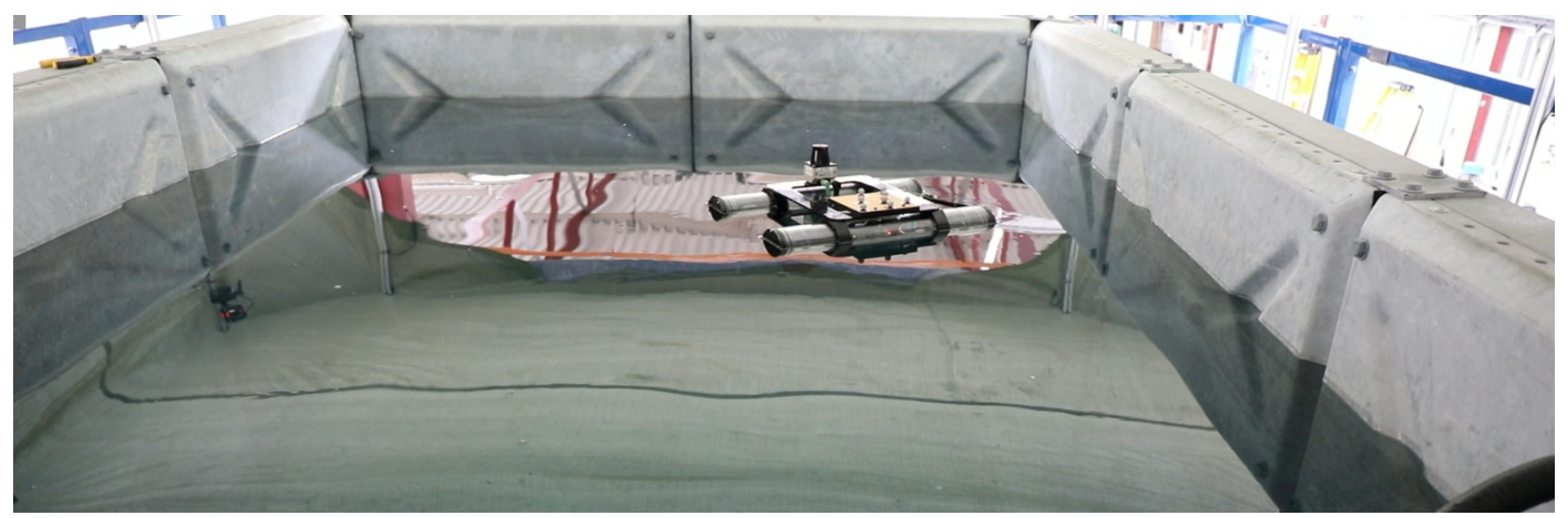

Fig. 1: MallARD in the testing pool that was used for conducting experiments.

\section{B. Thrust allocation}

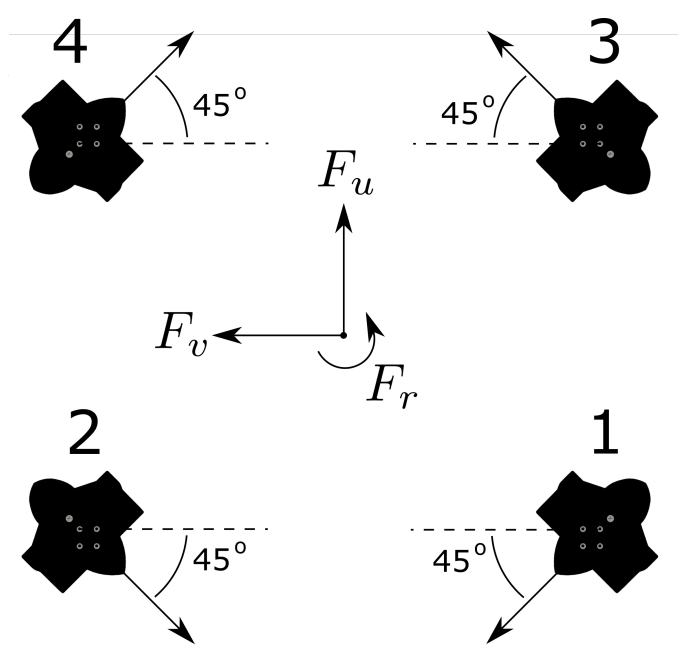

Fig. 2: MallARD's thruster layout in relation to its control inputs.

The thruster layout of MallARD is presented in Figure 2. Throughout this work the always forward (AF) thrust allocation scheme presented in [8] is used. This scheme eliminates the problems associated with deadbands by ensuring that all thrusters are always spinning clockwise and never change direction. When there is no control input, all four thrusters spin clockwise with the same low level of thrust, which due to the symmetric layout of the thrusters generates no net thrust. To move forward, for example, inputs to thrusters 3 and 4 are increased, while no change is made to the level of thrust delivered by thrusters 1 and 2. To rotate clockwise, inputs to thrusters 1 and 4 are increased. Movement that involves inputs in more than one degree of freedom is treated as a linear combination of the thrusters inputs for each of the degrees of freedom. Full detail of the AF thrust allocation scheme is described in [8].

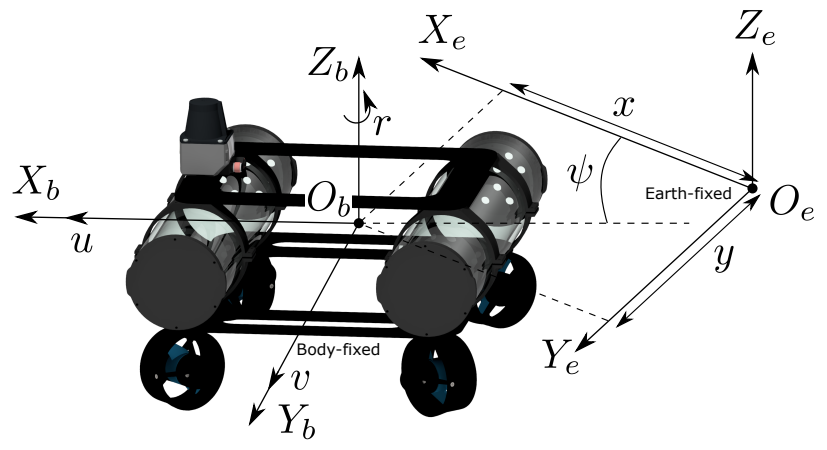

Fig. 3: Schematic diagram showing the choice of earth and body reference frames for MallARD.

\section{DYNAMIC MODEL}

\section{A. Kinematics and reference frames}

Due to its mechanical design, MallARD is stable in roll and pitch. Wave disturbance is small in the pool and for this reason motions in pitch, roll, and heave are neglected in this work. Therefore, the general six degrees of freedom model used for aquatic vehicles [20] can be simplified to three DOF.

Figure 3 presents the coordinate system and reference frames used in this work. The yaw angle is represented by $\psi$ and the robot coordinates in $X_{e}$ and $Y_{e}$ by $x$ and $y$, respectively. The coordinate vector in the earth-fixed frame is $\boldsymbol{\eta}=[x, y, \psi]^{\mathrm{T}}$, and the velocity vector becomes $\dot{\boldsymbol{\eta}}=$ $[\dot{x}, \dot{y}, \dot{\psi}]^{\mathrm{T}}$. The body frame velocities in $X_{b}$ and $Y_{b}$ are $u$ and $v$, while the angular velocity is $r$. The velocity vector is $\boldsymbol{\nu}=[u, v, r]^{\mathrm{T}}$ and the relationship between the body frame velocity vector and the earth-fixed velocity vector is:

$$
\dot{\boldsymbol{\eta}}=\boldsymbol{R}(\psi) \boldsymbol{\nu}
$$

where the frame rotation matrix is given by:

$$
\boldsymbol{R}(\psi)=\left[\begin{array}{ccc}
\cos \psi & -\sin \psi & 0 \\
\sin \psi & \cos \psi & 0 \\
0 & 0 & 1
\end{array}\right]
$$




\section{B. Dynamics}

Following Newton's second law of motion and the mainstream conventions (e.g. [11] and [21]) a dynamic model of MallARD in the body frame can be expressed as:

$$
M \dot{\nu}+C(\nu) \nu+D(\nu) \nu=\tau,
$$

where,

$$
\begin{aligned}
& \boldsymbol{M}=\left[\begin{array}{ccc}
m & 0 & 0 \\
0 & m & 0 \\
0 & 0 & I_{z}
\end{array}\right] \\
& \boldsymbol{C}(\boldsymbol{\nu})=\left[\begin{array}{ccc}
0 & 0 & -m v \\
0 & 0 & m u \\
0 & 0 & 0
\end{array}\right] \\
& \boldsymbol{D}(\boldsymbol{\nu})=\boldsymbol{D}+\boldsymbol{D}_{N}(\boldsymbol{\nu}) \text {, } \\
& \boldsymbol{D}=-\left[\begin{array}{ccc}
X_{u} & 0 & 0 \\
0 & Y_{v} & 0 \\
0 & 0 & N_{r}
\end{array}\right] \\
& \boldsymbol{D}_{N}(\boldsymbol{\nu})=\left[\begin{array}{ccc}
-X_{|u| u}|u| & 0 & 0 \\
0 & -Y_{|v| v}|v| & 0 \\
0 & 0 & -N_{|r| r}|r|
\end{array}\right] \text {, } \\
& \boldsymbol{\tau}=\left[\begin{array}{c}
\tau_{u} \\
\tau_{v} \\
\tau_{r}
\end{array}\right]=\left[\begin{array}{c}
X_{F} F_{u} \\
Y_{F} F_{v} \\
N_{F} F_{r}
\end{array}\right]
\end{aligned}
$$

$M$ is the mass matrix with $m_{x}, m_{y}$ and $I_{z}$ being the total mass in $x$, total mass in $y$ and moment of inertia around $Z_{b}$, respectively. It is assumed that the added mass in $x$ and $y$ is constant. The vector of centripetal forces is represented by $\boldsymbol{C}(\boldsymbol{\nu}) \boldsymbol{\nu}$, the drag forces vector is $\boldsymbol{D}(\boldsymbol{\nu}) \boldsymbol{\nu}$ and $\tau$ is the vector of external forces and torques. The linear drag coefficients are $X_{u}, Y_{v}, N_{r}$, while the non-linear drag coefficients are $X_{|u| u}, Y_{|v| v}, N_{|r| r}$. The force inputs to the system are $F_{u}, F_{v}, F_{r}$ with $X_{F}, Y_{F}, N_{F}$ being the associated linear force coefficients. More detailed description of the choice of parameters can be found in [8]. In this paper some of the coupling terms presented in [8] are omitted, because they have negligible effect on the measured accuracy of the model.

\section{EXPERIMENTS AND DATA COLLECTION}

Data for the system identification of MallARD was collected in a pool that was $2.4 \mathrm{~m}$ wide and $3.6 \mathrm{~m}$ long, shown in Figure 1 .

In total, 12 datasets were recorded in three repeat experiments using four different motion patterns. Each dataset was between 5 and 6 minutes in length. For the first three datasets $S_{1}, S_{2}$ and $S_{3}$, MallARD was predominantly excited in $X_{b}$ (Figure 3) by control inputs in $F_{u}$ (Figure 2). In the second three datasets $S_{4}, S_{5}, S_{6}$ and third three datasets, $S_{7}, S_{8}, S_{9}$, excitation was focused on $Y_{b}$ and $\psi$ by $F_{v}$ and $F_{r}$, respectively. For datasets $S_{1}$ to $S_{9}$ excitation was induced by pulsing the joypad on a single axis. The period and amplitude of the pulses was varied so that a range of accelerations and velocities were generated. In the final three datasets $S_{10}, S_{11}, S_{12}$, MallARD was excited in all degrees of freedom and was driven in squares, circles and figures of eight, which are motions that could be expected in practice. Throughout the experiments, the driver kept the robot at least $0.5 \mathrm{~m}$ away from the walls, because if the thrusters do not have open water surrounding them their performance can be affected. Camera footage showing sections of the data collection can be viewed in the video accompaniment to this paper.

The datasets consisted of both recorded joystick control inputs and earth fixed frame coordinates, obtained from the SLAM algorithm output. The joystick generated data at a rate of $100 \mathrm{~Hz}$, while the pose data was created at $15 \mathrm{~Hz}$. The datasets were interpolated so that data points were synchronised at a fixed rate of $15 \mathrm{~Hz}$. Earth-fixed velocities were estimated at each point using the five-point stencil method and body frame velocities were then calculated using Equation 1.

\section{MODEL IDENTIFICATION}

\section{A. Parametric model identification using least squares fitting}

To prepare the parametric model for identification, Equations 3 to 9 in Section III were rearranged as follows with lumped coefficients:

$$
\begin{aligned}
& \dot{u}=v r+\left(X_{|u| u c}|u|+X_{u c}\right) u+X_{F c} F_{u} \\
& \dot{v}=-u r+\left(Y_{|v| v c}|v|+Y_{v c}\right) v+Y_{F c} F_{v} \\
& \dot{r}=\left(N_{|r| r c}|r|+N_{r c}\right) r+N_{F c} F_{r}
\end{aligned}
$$

where $X_{|u| u c}=\frac{X_{|u| u}}{m}, X_{u c}=\frac{X_{u}}{m}, X_{F c}=\frac{X_{F}}{m}, Y_{|v| v c}=$ $\frac{Y_{|v| v}}{m}, Y_{v c}=\frac{Y_{v}}{m}, Y_{F c}=\frac{Y_{F}}{m}, N_{|r| r c}=\frac{N_{|r| r}}{I_{z}}, N_{r c}=\frac{N_{r}}{I_{z}}$, $N_{F c}=\frac{N_{F}}{I_{z}}$. It was assumed that the added mass in $X_{b}$ and $Y_{b}$ are of equal magnitude, because the robot moves with low velocity and acceleration. Equation 10 was discretised so that coefficients could be determined using the least squares method. Datasets $S_{1}, S_{2}, S_{4}, S_{5}, S_{7}, S_{8}, S_{10}$ and $S_{11}$ were used in the identification procedure, while datasets $S_{3}$ (excitation in $\left.X_{b}\right)$ ), $S_{6}$ (excitation in $Y_{b}$ ), $S_{9}$ (excitation in $\psi$ ) ) and $S_{12}$ (combined motion) were set aside for testing. Detail of the procedure used to identify coefficients can be found in [8] but is omitted here for brevity.

\section{B. LSTM network}

An LSTM recurrent neural network was also used to identify the dynamic system model. Since the dynamic behaviour of MallARD is temporally sensitive and depends not only on present inputs, but also on previous inputs and outputs, the LSTM architecture was chosen as it is able to capture temporal dynamics.

Our network architecture is shown in Figure 4, with the structure of an individual cell is presented in Figure 5 . The three input vectors to the cell are $c_{t-1}, h_{t-1}$ and $x_{t}$, where $x_{t}$ is a $1 \times 6$ vector with three of the columns containing the force inputs $F_{x}, F_{y}$ and $F_{z}$ and the other three being the body frame velocities $u_{t}, v_{t}$ and $r_{t}$. The output from each cell is $h_{t}$ and $c_{t}$ is the memory control variable that is passed to the next cell. Each sigmoid and hyperbolic tangent layer 


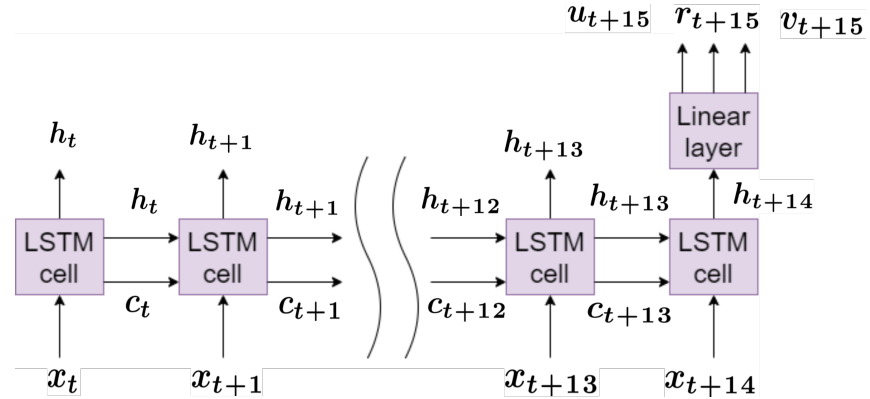

Fig. 4: Schematic of the full LSTM recurrent neural network used to model robot's dynamic behaviour.

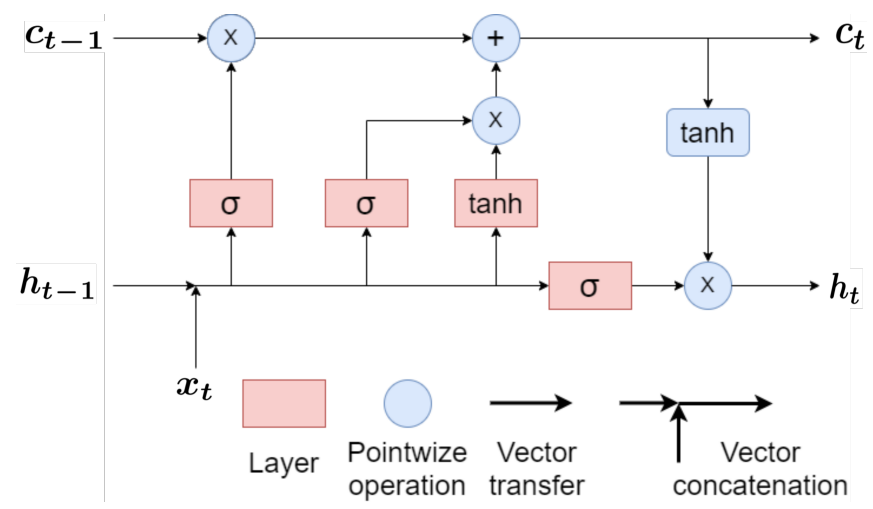

Fig. 5: A single cell of the LSTM network, where $x_{t}$ is the input at the current time step, $h_{t}$ is the output at the current time step and $c_{t}$ are values passed between the LSTM cells acting as memory control.

Figure 5] was comprised of 50 neurons. The network was trained to predict the output data using fifteen timesteps, therefore fifteen stacked LSTM cells were used, predicting velocities in $\left(X_{b}\right),\left(Y_{b}\right)$ and $(\psi)$ at timestep $t$, using previous velocities and force inputs from timestep $t-15$ to $t-1$. The network was tested with the number of cells ranging from 2 to 30 and error minimisation converged at 15 cells.

A layer of three linear output neurons provided the model output (body frame velocities in 3 degrees-of-freedom). Datasets used for training the network were the same as those used for identification of the parametric model. A variety of cost functions and batch sizes were explored. Best results were achieved using a mean squared error cost function and a batch size of 1024. The network was trained with 20 epochs using the ADAM training optimizer [22], which provided heightened performance compared to other optimisers (e.g., RMSprop [23]) during initial testing.

\section{RESULTS AND DISCUSSION}

\section{A. Single axis excitation}

The main purpose of the dynamic model is to allow both software and hardware simulation of MallARD's dynamic behaviour. Therefore, the two dynamic models were tested based upon their ability to predict velocities over a long time horizon, using only initial conditions, i.e. an infinite step-ahead model. The efficacy of the LSTM network is

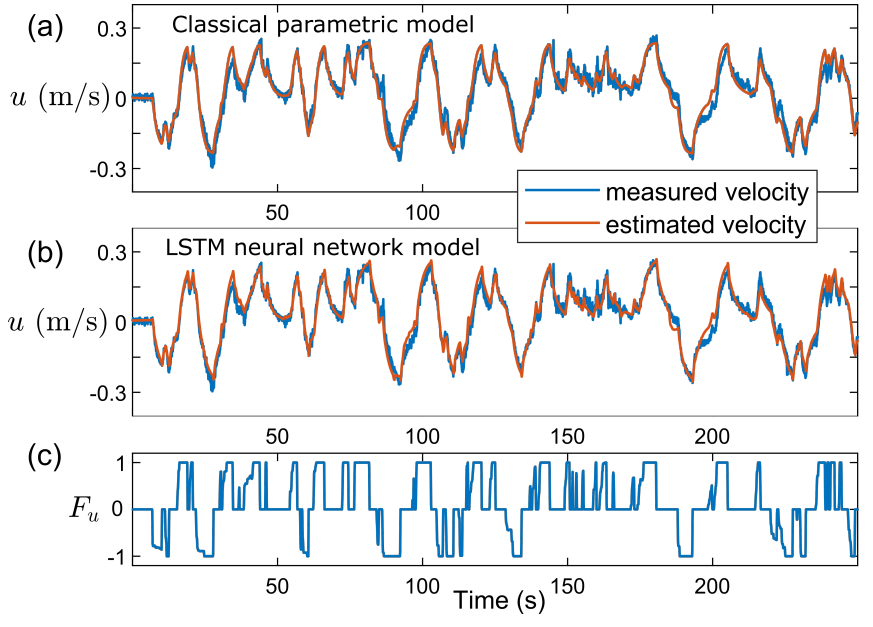

Fig. 6: Plots (a) and (b) show the measured velocity $u$ in $X_{b}$ taken from dataset $S_{3}$ against the estimated velocity in $X_{b}$ for the classical parametric model and the LSTM model respectively; Plot (c) displays the control input $F_{u}$.

compared against the classical parametric dynamic model, and accompanying coefficient identification procedure, that was previously developed by the authors. The datasets used for testing the dynamic models were previously unseen datasets $S_{3}, S_{6}, S_{9}$ and $S_{12}$. These datasets were not used in any of the training or identification procedures.

Considering dataset $S_{3}$, where the inputs from the joystick were predominantly in $F_{u}$, Figure 6 shows that both the classical parametric model and the LSTM network perform well and with similar error. Table I] presents the RMS error between the estimated and the actual velocities $u, v$ and $r$ for the respective $S_{3}, S_{6}$ and $S_{9}$ datasets. These results show that the LSTM network performs slightly better overall when excitation is predominantly in a single degree-of-freedom and velocity predictions are made on the same axis. However, it was noticed during experiments that when the robot was excited in $F_{u}$ (on $X_{b}$ ), MallARD began to rotate, indicating coupling between linear motion in $X_{b}$ and rotation about $Z_{b}$. Attempts to model this coupling by adding various parameters to the parametric model was unsuccessful but the LSTM model was able to model this coupling effect without modification. This can be observed in Figure 7, where estimated and actual yaw velocities are plotted for both dynamic models for dataset $S_{3}$. In dataset $S_{3}$, excitation was predominantly in $F_{u}$; excitation in $F_{r}$ was only used to correct the robot's orientation. In the time region between $90 \mathrm{~s}$ and $140 \mathrm{~s}$ (Figure 7), there was no excitation in $F_{r}$ but there was induced velocity in $r$ due to excitation in $F_{u}$ and the LSTM network clearly outperforms the parametric model in this region.

\section{B. Multi-axis excitation}

The improvements in model accuracy of the LSTM network when compared to the classical model is more prominent when tested on the combined motion dataset $\left(S_{12}\right)$. Figure 8 shows the LSTM model estimates tracking the real $v$ 
TABLE I: RMS errors between predicted velocities and measured velocities.

\begin{tabular}{cccc}
\hline $\begin{array}{c}\text { Body Frame } \\
\text { Velocity }\end{array}$ & Dataset & LSTM & Classical Model \\
\hline \hline$u$ & $S_{3}$ & $0.026 \mathrm{~m} / \mathrm{s}$ & $0.032 \mathrm{~m} / \mathrm{s}$ \\
\hline$v$ & $S_{6}$ & $0.027 \mathrm{~m} / \mathrm{s}$ & $0.026 \mathrm{~m} / \mathrm{s}$ \\
\hline$r$ & $S_{9}$ & $0.038 \mathrm{rad} / \mathrm{s}$ & $0.062 \mathrm{rad} / \mathrm{s}$ \\
\hline
\end{tabular}

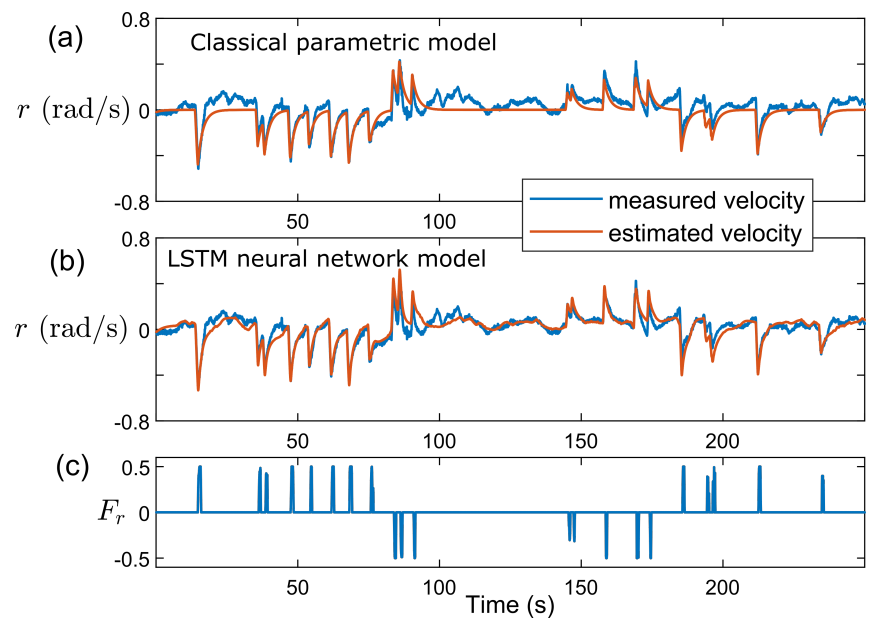

Fig. 7: Plots (a) and (b) show the measured angular velocity $r$ about $Z_{b}$ taken from dataset $S_{3}$ against the estimated velocity for the classical parametric model and the LSTM model respectively; Plot (c) displays the control input $F_{r}$.

velocities much closer than the parametric model. Similar results were derived for $u$ and $r$ using the $S_{12}$ dataset. This is evident in the histograms that are presented in Figure 9 The histograms presenting error from the LSTM network show consistently lower mean error and lower deviation of errors than the histograms associated with the classical parametric model.

\section{CONCLUSIONS}

An LSTM recurrent neural network has proven to be a useful method for generating a system model that can reproduce the temporal-dynamics of an aquatic vehicle. The LSTM model developed in this paper was demonstrated to be capable of producing accurate and reliable velocity estimates over long time periods ( $250 \mathrm{~s}$ ) when provided with the vehicle's control inputs. When compared directly against a classically derived parametric model, the LSTM model showed clear improvements in accuracy, particularly in the more realistic case of control inputs in multiple degrees of freedom. The LSTM network reduced the RMS velocity estimation error by an average (mean) of $35.67 \%$ compared to the classical parametric model, in the more realistic case of control inputs across all three degrees-of-freedom.

The neural network also requires less manual setup; this makes it both faster to deploy and less prone to errors caused by the selection of equation terms.
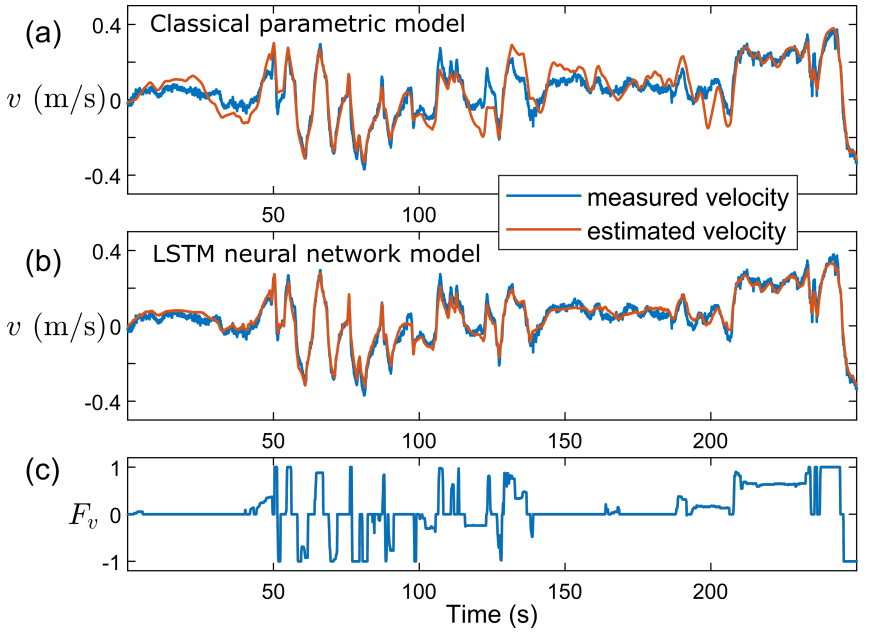

Fig. 8: Plots (a) and (b) show the measured velocity $v$ in $Y_{b}$ taken from dataset $S_{12}$ against the estimated velocity in $Y_{b}$ for the classical parametric model and the LSTM model respectively; Plot (c) displays the control input $F_{v}$.
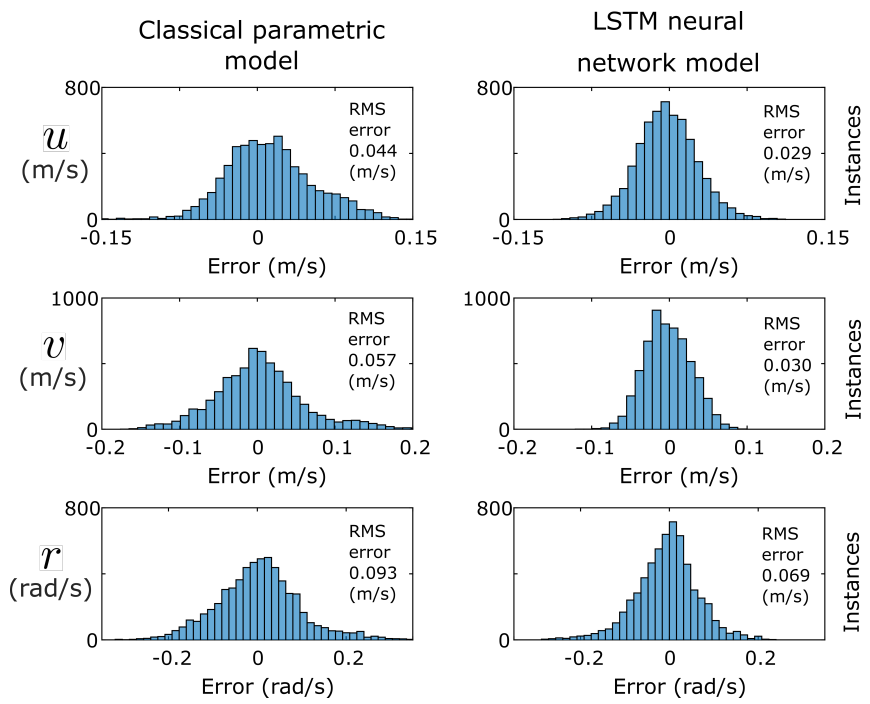

Fig. 9: Histograms presenting the prediction error of the two different models for velocities $u$ in $X_{b}, v$ in $Y_{b}$ and $r$ about $Z_{b}$ for the $S_{12}$ dataset.

The trained LSTM neural network is presently being used to facilitate research on the MallARD ASV in both software and hardware simulations (see Section II-A.

\section{ACKNOWLEDGMENT}

This research was funded by the Engineering and Physical Sciences Research Council under grant: EP/P01366X/1

\section{REFERENCES}

[1] M. Dunbabin and A. Grinham, "Experimental evaluation of an autonomous surface vehicle for water quality and greenhouse gas emission monitoring," in 2010 IEEE International Conference on Robotics and Automation, pp. 5268-5274, IEEE, 2010.

[2] K. Groves, A. West, K. Gornicki, S. Watson, J. Carrasco, and B. Lennox, "Mallard: An autonomous aquatic surface vehicle for inspection and monitoring of wet nuclear storage facilities," Robotics, vol. 8, no. 2, p. 47, 2019. 
[3] L. Pitonakova, M. Giuliani, A. Pipe, and A. Winfield, "Feature and performance comparison of the v-rep, gazebo and argos robot simulators," in Annual Conference Towards Autonomous Robotic Systems, pp. 357-368, Springer, 2018.

[4] C. Lennox, K. Groves, V. Hondru, F. Arvin, K. Gornicki, and B. Lennox, "Embodiment of an aquatic surface vehicle in an omnidirectional ground robot," in 2019 IEEE International Conference on Mechatronics (ICM), vol. 1, pp. 182-186, IEEE, 2019.

[5] S. Arnold and L. Medagoda, "Robust model-aided inertial localization for autonomous underwater vehicles," in 2018 IEEE International Conference on Robotics and Automation (ICRA), pp. 1-9, IEEE, 2018.

[6] Ø. Hegrenaes and O. Hallingstad, "Model-aided ins with sea current estimation for robust underwater navigation," IEEE Journal of Oceanic Engineering, vol. 36, no. 2, pp. 316-337, 2011.

[7] S. Watson, D. A. Duecker, and K. Groves, "Localisation of unmanned underwater vehicles (UUVs) in complex and confined environments: A review," Sensors, vol. 20, no. 21, p. 6203, 2020.

[8] K. Groves, M. Dimitrov, H. Peel, O. Marjanovic, and B. Lennox, "Model identification of a small omnidirectional aquatic surface vehicle: a practical implementation," in Intelligent Robots and Systems, 2020. IROS 2020., IEEE, 2020.

[9] B. Allotta, R. Costanzi, L. Pugi, and A. Ridolfi, "Identification of the main hydrodynamic parameters of typhoon auv from a reduced experimental dataset," Ocean Engineering, vol. 147, pp. 77 - 88, 2018.

[10] B.-O. H. Eriksen and M. Breivik, "Modeling, identification and control of high-speed asvs: Theory and experiments," in Sensing and Control for Autonomous Vehicles, pp. 407-431, Springer, 2017.

[11] C. Li, J. Jiang, F. Duan, W. Liu, X. Wang, L. Bu, Z. Sun, and G. Yang, "Modeling and experimental testing of an unmanned surface vehicle with rudderless double thrusters," Sensors, vol. 19, no. 9, p. 2051, 2019.

[12] C. R. Sonnenburg and C. A. Woolsey, "Modeling, identification, and control of an unmanned surface vehicle," Journal of Field Robotics, vol. 30, no. 3, pp. 371-398, 2013.

[13] J. Woo, J. Park, C. Yu, and N. Kim, "Dynamic model identification of unmanned surface vehicles using deep learning network," Applied Ocean Research, vol. 78, pp. 123-133, 2018.

[14] K. Groves and P. Bonello, "Empirical identification of squeeze-film damper bearings using neural networks," Mechanical Systems and Signal Processing, vol. 35, no. 1-2, pp. 307-323, 2013.

[15] J. Hwangbo, J. Lee, A. Dosovitskiy, D. Bellicoso, V. Tsounis, V. Koltun, and M. Hutter, "Learning agile and dynamic motor skills for legged robots," Science Robotics, vol. 4, no. 26, 2019.

[16] A. Punjani and P. Abbeel, "Deep learning helicopter dynamics models," in 2015 IEEE International Conference on Robotics and Automation (ICRA), pp. 3223-3230, 2015.

[17] C. Andersson, A. H. Ribeiro, K. Tiels, N. Wahlström, and T. B. Schön, "Deep convolutional networks in system identification," in 2019 IEEE 58th Conference on Decision and Control (CDC), pp. 3670-3676, 2019.

[18] E. de la Rosa and W. Yu, "Randomized algorithms for nonlinear system identification with deep learning modification," Information Sciences, vol. 364-365, pp. 197 - 212, 2016.

[19] S. Kohlbrecher, O. Von Stryk, J. Meyer, and U. Klingauf, "A flexible and scalable slam system with full 3d motion estimation," in 2011 IEEE international symposium on safety, security, and rescue robotics, pp. 155-160, IEEE, 2011.

[20] T. I. Fossen, "Guidance and control of ocean vehicles," University of Trondheim, Norway, Printed by John Wiley \& Sons, Chichester, England, ISBN: 047194113 1, Doctors Thesis, 1999.

[21] R. Skjetne, Ø. N. Smogeli, and T. I. Fossen, "A nonlinear ship manoeuvering model: Identification and adaptive control with experiments for a model ship," 2004.

[22] D. P. Kingma and J. Ba, "Adam: A method for stochastic optimization," arXiv preprint arXiv:1412.6980, 2014.

[23] S. Ruder, "An overview of gradient descent optimization algorithms," arXiv preprint arXiv:1609.04747, 2016. 